\title{
Dysmenorrhoea and Adnexal Cysts - A Rare Presentation of Tarlov Cysts in the Pelvis
}

\author{
Eric Tat Choi Lee and Felix Wu Shun Wong* \\ Consultants, O\&G unit, Canossa Hospital, Hong Kong
}

\begin{abstract}
Tarlov cysts are meningeal dilations of the posterior spinal nerve root. The cysts can grow as a result of an increased inflow of cerebrospinal fluid giving rise to symptoms due to compression or stretching of the adjacent nerve roots. They are usually multiple and asymptomatic, but Tarlov cysts in the sacral neural canal and foramina can be associated with symptoms like radicular pain, low back pain, paresthesia, and urinary or bowel dysfunction. To the best of our knowledge, Tarlov cysts presented with dysmenorrhea has not been reported in the literature. This association, though rare, should not be overlooked, especially when there is enough clinical suspicion while investigating an atypically looking adnexal cyst while managing in the context of dysmenorrhea or pelvic pain. MRI is recommended when the exact diagnosis is in doubt. Laparoscopic excision of the cyst is feasible and in our case it has been shown to be effective and safe.
\end{abstract}

Keywords: Tarlov cyst; Dysmenorrhea; Pelvic pain; Laparoscopic excision

\section{Case report}

A 41 year old, married for 20 years, presented on $28^{\text {th }}$ May, 2013 with history of dysmenorrhoea, deep dyspareunia, and pelvic pain for the past 6 months. She was nulliparous and practiced contraception by using condoms. Her menstrual pattern was essentially normal and the pain occurred mainly on the first two days of her period. Initial vaginal ultrasound scanning revealed multiple adnexal cysts on both sides of the pelvis (Figure 1a) with the largest one measured at $5 \mathrm{~cm}$ on the left. Bimanual pelvic examination elicited tenderness over the uterosacral ligaments and demonstrated the presence of the cystic adnexal masses. The initial diagnosis was probable ovarian endometrioma and pelvic endometriosis. Laparoscopy was performed with a view to excise any endometriotic lesions and to perform ovarian cystectomy on $1^{\text {st }}$ June, 2013. Except for a small left ovarian dermoid that was removed at the operation, the uterus, and tubes, right ovary and uteroscral ligaments

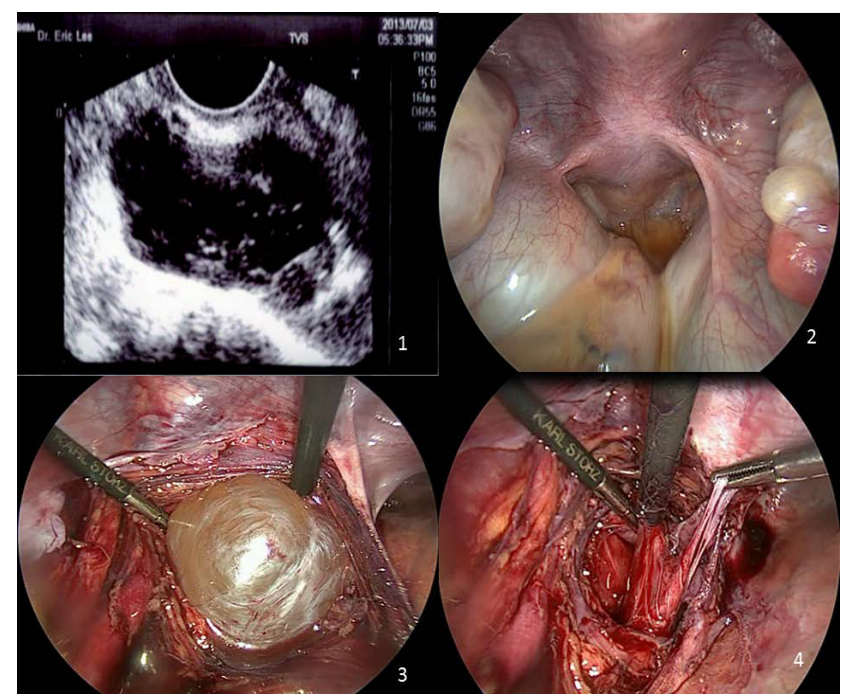

Figure 1: (a) Vaginal ultrasound scanning of pelvis showing multiple adnexal cysts with a large multilobuated right adnexal cyst. (b): Laparoscopic view of Tarlov cysts in the pelvis. (c): Exposure of the Tarlov cyst wall following dissection. (d): Tarlov cyst wall attached to the nerve bundle lying on the pelvic floor. were grossly normal but there was no evidence of any endometriosis. On the contrary, bilateral retroperitoneal cystic swellings were seen lying underneath the uterosacral ligaments and rested against the pelvic floor on both sides (Figure 1b). The cyst on the left side was more prominent and dissection was carried out to completely isolate the cyst after the ureter was lateralized and the rectum deflected to the opposite side using the rectal probe. The cyst was thin walled, smooth, containing clear fluid, and fibrous in nature (Figure 1c). A pedicle was found connecting the cyst to a cystic tubular neurogenic-like structure that lied across the pelvic floor (Figure 1d). The cyst was excised after its rupture with the release of a lightly yellowish clear fluid. The inner wall of the cyst was smooth with no communicating channels identifiable. No further procedure was performed in view of the uncertainty of the pathology involved. The immediate postoperative recovery was uneventful and the patient was discharged home on the first postoperative day. She returned in a week for assessment and reported shooting pain over the dermatomes corresponding to S2 and S3 of the sacral nerves on the left side, starting two days following the surgery. MRI scanning to the pelvis was subsequently performed and revealed features consistent with type 2 Tarlov cysts involving sacral nerves from S1 to S3 on both sides (Figure 2a-2c). On pathological examination, the specimen grossly consisted of a $3 \times 1.5 \times 1 \mathrm{~cm}$ irregular pale tan soft tissue weighing $1 \mathrm{~g}$. The soft tissue appeared to form the cyst wall with no other remarkable features. On microscopic examination, the tissue was composed of mostly bland-appearing fibrous spindle cells with no definitive epithelial or mesothelial cells lining. . In some areas, small to medium-sized fascicles of spindle cells were present, suggestive of nerve fibres. There was no evidence of significant inflammation or tumour. The tissue underwent immunostaining which showed positive staining for S-100 protein, a marker of neural tissue (Figure 2d). On the other hand, cytokeratin (a marker for epithelial cells, including

${ }^{*}$ Corresponding author: Felix Wu Shun Wong, Consultant, O\&G unit, Canossa Hospital, Hong Kong, E-mail: fwong3@hotmail.com.hk

Received January 05, 2015; Accepted January 26, 2015; Published January 28 2015

Citation: Lee ETC, Wong FWS (2015) Dysmenorrhoea and Adnexal Cysts - A Rare Presentation of Tarlov Cysts in the Pelvis. J Clin Case Rep 5: 476. doi:10.4172/2165-7920.1000476

Copyright: @ 2015 Lee ETC, et al. This is an open-access article distributed under the terms of the Creative Commons Attribution License, which permits unrestricted use, distribution, and reproduction in any medium, provided the original author and source are credited. 


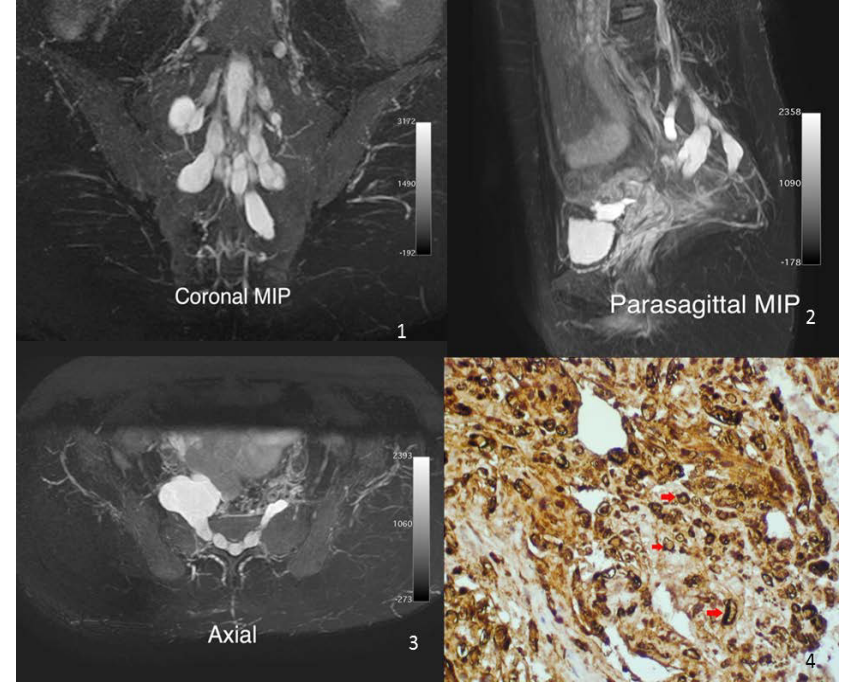

Figure 2: MRI of the pelvis (a) coronal, (b) left parasagittal, (c) axial views after the surgery (d) - the immunohistochemical staining with S-100 protein. The small darkly stained ring-shaped structures (red arrows) are myelinated nerves $(\mathrm{S}-100 \times 400)$

mesonephric and Wolffian duct system epithelial cells) and Calretinin (a marker for mesothelial cells) were negative on the cyst surface. The staining pattern confirmed the presence of neural elements (nerve fibres) in the cyst wall, therefore supported the diagnosis of Tarlov cyst instead of epithelial or mesothelial cysts. She was then referred to a neurologist who treated her symptomatically with analgesic. Her pain over the thigh gradually subsided and she reported complete remission on $3^{\text {rd }}$ July, 2013, 6 weeks postoperatively. She returned for regular follow up on $18^{\text {th }}$ December, 2014 and reported persistence of mild pelvic discomfort despite improvement of dysmenorrhoea. Repeated ultrasound scanning still showed multiple pelvic cysts.

\section{Discussion}

Tarlov cysts are estimated to affect 4.6 to $9 \%$ of the adult population $[1,2]$. The exact etiology remains unclear. They are pathological formations located at the junction of the dorsal root ganglion and its nerve root, arising between the arachnoid covering (perineurium) and the pia (endoneurium). There are nerves fibres present along the lining of these lesions. They are mostly asymptomatic and may only be incidental findings on routine gynecological ultrasound examination. They are often multiple and assume a tubular/cystic or multilocular/ multiseptate appearance. Commonly reported symptoms associated with Tarlov cysts include low back pain, sciatica, genitourinary or bowel dysfunction, and abdominal pain [3-5]. Saboo and Di Salvo 2013 reported a case of perineural cysts discovered incidentally on pelvic sonography on a 54 year old lady who had a history of stage 1 breast cancer and presented with postmenopausal spotting with the findings of suspicious complex cystic adnexal masses. In the context of the clinical history, both CT and MRI were performed, and fortunately they revealed the finding of perineural cysts prior to operation [6]. Dysmenorrhoea, a gynaecological complaint, has so far not been reported in the literature as one of the related symptoms as reported here. In the pelvis, they can take the form of a retroperitoneal cyst. Differential diagnoses of other retroperitoneal pelvic cysts include those of mesothelial, mesonephric, or paramesonephric in origin. Detailed ultrasound scanning may provide a clue to the correct diagnosis. Sonographic features reported of perineural cysts include (1) multiseptated complex cysts with internal debris, which may be separate from the ovaries; (2) posterior location in the pelvis, adjacent to the sacrum; (3) lack of mobility due to their extraperitoneal location [7-9]. If the clinical diagnosis is in doubt, magnetic resonance imaging should be used to confirm the diagnosis. Ghazizadeh, Foss et al. in their review of CT, MRI and ultrasound imaging of various pseudotumours in the pelvis, raised the awareness of pitfalls to diagnose perineural cyst and thus avoiding a misdiagnosis and any unnecessary surgical intervention [10]. Most Tarlov cysts do not require any treatment as they are usually asymptomatic. However, treatment may be indicated when the symptom becomes progressive and affects one's quality of life. In our case, preoperative diagnosis of pelvic endometriosis and bilateral ovarian cysts were made on clinical ground and ultrasound scan. As a result, CT or MRI scans had not been considered due to the misdiagnosis of endometriosis related dysmenorrhoea and the expensive cost of CT/MRI incurred in private practice. Laparoscopy was performed with a view to providing the necessary treatment. Laparoscopic excision of Tarlov cyst was feasible as demonstrated in our case, though other techniques like incision and drainage without excision, cyst fenestration and imbrication have been proposed $[1,11]$. While dysmenorrhea disappeared after the excision of the Tarlov cyst in our patient, we believe that release of the pressure exerted by the large cyst on the neural tissue could account for the favourable outcome.

\section{References}

1. Paulsen RD, Call GA, Murtagh FR (1994) Prevalence and percutaneous drainage of cysts of the sacral nerve root sheath (Tarlov cysts). AJNR Am J Neuroradiol 15: 293-297.

2. Smith DT (2003) Cystic formations associated with human spinal nerve roots Journal of Neurosurgery, 18: 654-660.

3. Ishii K, Yuzurihara M, Asamoto S, Doi H, Kubota M (2007) A huge presacral Tarlov cyst. Case report. J Neurosurg Spine 7: 259-263.

4. Slipman CW, Bhat AL, Bhagia SM, Issac Z, Gilchrist RV, et al. (2003) Abdominal pain secondary to a sacral perineural cyst. Spine J 3: 317-320.

5. Caspar W, Papavero L, Nabhan A, Loew C, Ahlhelm F (2003) Microsurgical excision of symptomatic sacral perineurial cysts: a study of 15 cases. Surg Neurol 59: 101-105.

6. Saboo SS, Di Salvo D (2013) Perineural cysts resembling complex cystic adnexal masses on transvaginal sonography. J Clin Ultrasound 41: 55-58.

7. McClure MJ, Atri M, Haider MA, Murphy J (2001) Perineural cysts presenting as complex adnexal cystic masses on transvaginal sonography. AJR Am J Roentgenol 177: 1313-1318.

8. H'ng $M$, Wanigasiri U, Ong $C$ : Perineural (Tarlov) cysts mimicking adnexal masses: a report of three cases. Ultrasound in Obstetrics \& Gynecology 2009, 34(2): 230-233.

9. Raza S, Klapholz H, Benacerraf BR (1994) Tarlov cysts: a cause of complex bilateral adnexal masses on pelvic sonography. J Ultrasound Med 13: 803-805.

10. Ghazizadeh S, Foss EW, Didier R, Fung A, Panicek DM, et al. (2014) Musculoskeletal pitfalls and pseudotumours in the pelvis: a pictorial review for body imagers. Br J Radiol 87: 20140243.

11. Mummaneni PV, Pitts LH, McCormack BM, Corroo JM, Weinstein PR (2000) Microsurgical treatment of symptomatic sacral Tarlov cysts. Neurosurgery 47 74-78. 